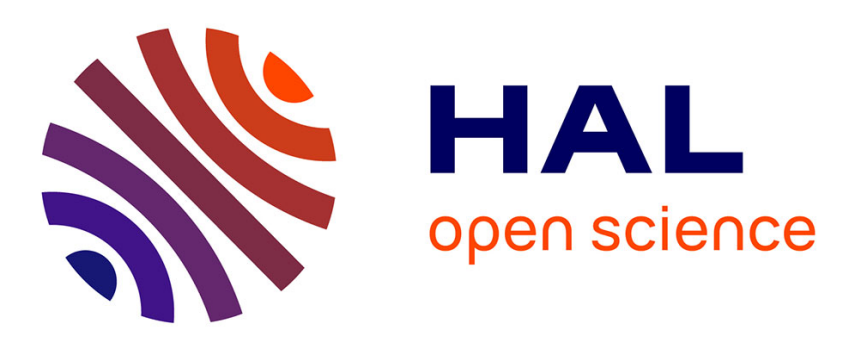

\title{
Hydrogen Plasma-Assisted Growth of Gold Nanowires
}

Ruiling Gong, Zhen Zheng, Junyang An, Jean-Luc Maurice, Edy Azrak, Vishnu Nair, Antonino Foti, Simona Moldovan, Chantal Karam, Sébastien Duguay, et al.

\section{- To cite this version:}

Ruiling Gong, Zhen Zheng, Junyang An, Jean-Luc Maurice, Edy Azrak, et al.. Hydrogen PlasmaAssisted Growth of Gold Nanowires. Crystal Growth \& Design, 2020, 20 (6), pp.4185-4192. 10.1021/acs.cgd.0c00480 . hal-03030277

\section{HAL Id: hal-03030277 https://hal.science/hal-03030277}

Submitted on 14 Dec 2020

HAL is a multi-disciplinary open access archive for the deposit and dissemination of scientific research documents, whether they are published or not. The documents may come from teaching and research institutions in France or abroad, or from public or private research centers.
L'archive ouverte pluridisciplinaire HAL, est destinée au dépôt et à la diffusion de documents scientifiques de niveau recherche, publiés ou non, émanant des établissements d'enseignement et de recherche français ou étrangers, des laboratoires publics ou privés. 
archives-ouvertes

\section{Hydrogen Plasma-Assisted Growth of Gold Nanowires}

Ruiling Gong, Zhen Zheng, Junyang An, Jean-Luc Maurice, Edy Azrak, Vishnu Nair, Antonino Foti, Simona Moldovan, Chantal Karam, Sébastien Duguay

\section{To cite this version:}

Ruiling Gong, Zhen Zheng, Junyang An, Jean-Luc Maurice, Edy Azrak, et al.. Hydrogen PlasmaAssisted Growth of Gold Nanowires. Crystal Growth and Design, American Chemical Society, 2020, 20 (6), pp.4185-4192. 10.1021/acs.cgd.0c00480 . hal-02988829

\section{HAL Id: hal-02988829 \\ https://hal.archives-ouvertes.fr/hal-02988829}

Submitted on 13 Nov 2020

HAL is a multi-disciplinary open access archive for the deposit and dissemination of scientific research documents, whether they are published or not. The documents may come from teaching and research institutions in France or abroad, or from public or private research centers.
L'archive ouverte pluridisciplinaire HAL, est destinée au dépôt et à la diffusion de documents scientifiques de niveau recherche, publiés ou non, émanant des établissements d'enseignement et de recherche français ou étrangers, des laboratoires publics ou privés. 


\section{Hydrogen plasma-assisted growth of gold nanowires}

Ruiling Gong ${ }^{1}$, Zhen Zheng ${ }^{1}$, Junyang $\mathrm{An}^{1}$, Jean-Luc Maurice ${ }^{2}$, Edy Azrak ${ }^{3}$, Vishnu Nair ${ }^{4}$, Antonino Foti $^{2}$, Simona Moldovan ${ }^{3}$, Chantal Karam $^{3}$, Sébastien Duguay ${ }^{3}$, Philippe Pareige ${ }^{3}$, Bozhi Tian ${ }^{4}$, Wanghua Chen ${ }^{1,2 *}$ and Pere Roca i Cabarrocas ${ }^{2 *}$

${ }^{1}$ School of Physical Science and Technology, Ningbo University, Ningbo 315211, China

${ }^{2}$ LPICM, CNRS, Ecole Polytechnique, Institut Polytechnique de Paris, 91128 Palaiseau, France

${ }^{3}$ GPM, Université et INSA de Rouen, CNRS, Normandie Université, Saint Etienne du Rouvray 76800, France

${ }^{4}$ Department of Chemistry, The University of Chicago, Chicago, Illinois 60637, United States

*E-mail: wanghua.chen@polytechnique.edu;

pere.roca@polytechnique.edu

Due to their innocuity, Au nanowires present an interesting field of applications in biology and, particularly, in cancer therapy. Since their morphology and distribution can greatly affect their performances, being able to control their mode of growth is important. Various elaboration techniques including "top-down" and "bottom-up" approaches have been developed. In this work, we propose an efficient maskless method to grow Au nanowires with the help of hydrogen plasma treatment of Au thin films. We have been able to grow $\mathrm{Au}$ nanowires by taking advantage of spinodal dewetting of an Au thin film and the supply of silicon radicals resulting from hydrogen plasma etching of amorphous silicon coating the walls of the reactor. A variety of techniques have been applied to study the microstructure and the optical properties of Au nanowires. A strong photothermal effect of Au nanowires has been demonstrated with the help of visible laser light. In order to study the nanowire growth, the transport of Au atoms is discussed and a growth mechanism is proposed. 


\section{Introduction}

After the discovery of the vapor-liquid-solid (VLS) growth mechanism of semiconductor nanowires (NWs) by Wagner and Ellis in $1964,{ }^{1}$ little attention was paid on NWs until Lieber's group reported the successful synthesis of crystalline Si NWs with diameters less than $20 \mathrm{~nm}$ in $1998 .^{2}$ Since then, many promising applications of NWs from different materials including semiconductors and metals have arisen. ${ }^{3-6}$ Au has been widely used as a catalyst to grow $\mathrm{Si} \mathrm{NWs}{ }^{7,8}$ or even to etch $\mathrm{Si}$ for mesostructures. ${ }^{9}$ In the case of metal NWs such as $\mathrm{Au} ; \mathrm{Ag}$ and $\mathrm{Cu}$, they have been widely used as transparent conductive materials for electrodes, for example, extensive research on the applications of Ag NWs for solar cells. ${ }^{10,11}$ As far as the application of Au NWs or nanorods, their use in biosensors such as DNA detectors ${ }^{12}$ and photothermal therapy of cancer $^{13,14}$ appears to be the most promising. The reason behind the application of Au NWs in biology can be explained by two facts. The first one is that $\mathrm{Au}$ is biodegradable, non-toxic and biologically inert. The second one is due to its unique electrical and optical properties, for example, its strong surface enhanced Raman scattering (SERS). ${ }^{15}$ Au NWs are an excellent candidate in photothermal systems such as ultrafast thermocycling ${ }^{16}$ and photothermal therapy (PTT), even with visible light.

To synthesize $\mathrm{Au}$ NWs, various methods have been developed. First, a "top-down" method using electron beam lithography (EBL) of an Au film. ${ }^{17}$ The second method is based on the chemical or electrochemical reduction of the template. ${ }^{18}$ This growth method includes a soft template and a hard template method. For the soft template method, Au NW growth process is chemically or electrochemically limited depending on the structure-directed molecules, which acts to inhibit the growth of certain crystal faces. For the hard template method, for example, anodized aluminum oxide (AAO) is used as a rigid template for a chemical reaction-based electrochemical growth. A third method is based on seed-mediated solution process, where Au NWs are grown from the Au seed in the solution. ${ }^{19}$ An extension 
of this solution method is a seedless approach, where the nucleation and growth of Au NWs occurs simultaneously. ${ }^{20}$ The last method is to fabricate Au NWs by depositing Au onto an epoxy substrate and then etching the epoxy resin. ${ }^{21,22}$

When considering the application of Au NWs, one of the most important issues is to control their arrangement. Since Au NWs are etched or grown with the help of a mask, the control of Au NWs arrangement can be achieved via the control of mask. Therefore, Au NWs elaboration is limited by the mask fabrication. In this work, we develop a maskless approach where the direct control of NW distribution is realized by the spinodal dewetting of $\mathrm{Au}$ droplets and Au adatom diffusion. A new Si source to help to grow Au NWs is exploited. Au NWs are synthesized in a plasma-enhanced chemical vapor deposition (PECVD) reactor using an Au thin film as a precursor. The advantages of PECVD-based growth method are its fast growth rate (hundreds of nanometers per minute) and large-scale production (up to several square meters). Multi-characterization techniques including scanning electron microscopy (SEM); transmission electron microscopy (TEM); energy dispersive X-ray (EDX); transmission Kikuchi diffraction (TKD); surface enhanced Raman scattering (SERS), and laser-induced photocurrent measurements are used to investigate the NWs morphology, their composition, and their optical properties.

\section{Results and discussion}

A schematic illustration of the fabrication process to grow maskless Au NWs is shown in Figure 1. The PECVD chamber is first pre-coated with hydrogenated amorphous $\mathrm{Si}(\mathrm{a}-\mathrm{Si}: \mathrm{H})$ at $120{ }^{\circ} \mathrm{C}$, with a thickness of $100 \mathrm{~nm}$. A c-Si (111) wafer is chosen as a substrate. The c-Si wafer is dipped into a HF solution (5\%) for 30 seconds in order to remove its native oxide, then $5 \mathrm{~nm}$ of Au thin film are thermally evaporated on it. The sample was then loaded into the PECVD reactor. Note that the PECVD reactor was purposely cooled down to room 
temperature in vacuum before opening the chamber in order to avoid the oxidation of a-Si:H. For the NW growth, only hydrogen (100 SCCM) is introduced into the PECVD reactor, the pressure is fixed at 1.33 mbar and the substrate temperature at $475{ }^{\circ} \mathrm{C}$ (calibrated temperature). Note that the substrate temperature here is calibrated by using Pt100 resistance thermometers. The plasma is ignited by applying a RF power and maintained for 10 minutes. During $\mathrm{H}_{2}$ plasma treatment, the influence of the plasma power was studied, while keeping other process conditions fixed.

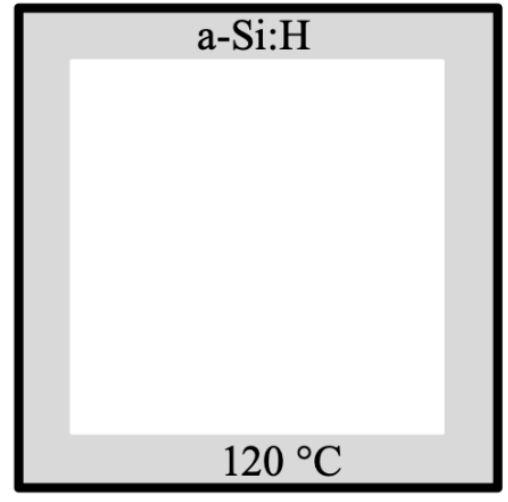

(a)

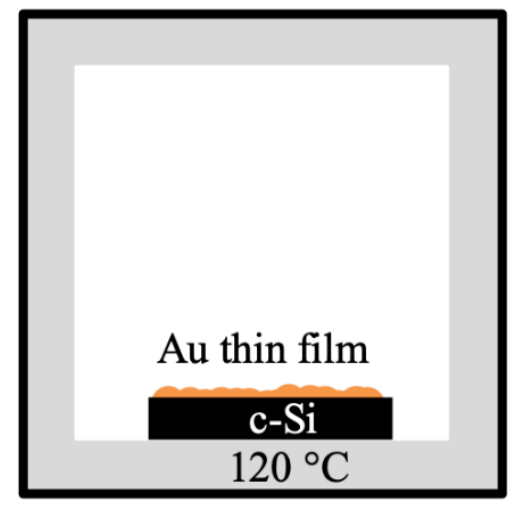

(b)

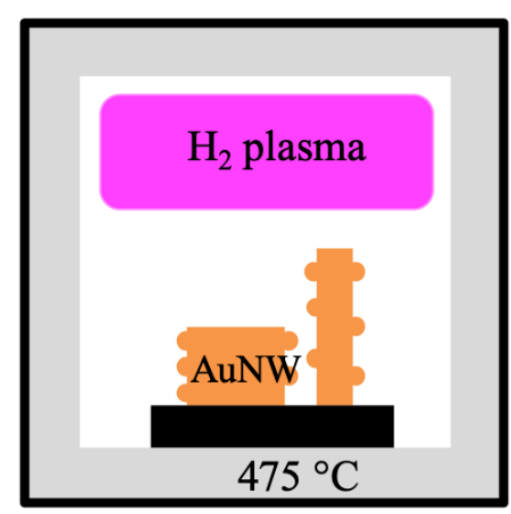

(c)

Figure 1. (a) Pre-coating of the PECVD chamber with a-Si:H at $120{ }^{\circ} \mathrm{C}$. (b) Loading a c-Si substrate covered with an Au thin film. (c) $\mathrm{H}_{2}$ plasma treatment of the sample at $475{ }^{\circ} \mathrm{C}$.

Figure 2 (a) and (b) present the corresponding top and side view SEM images of $\mathrm{Au}$ nanostructures obtained after 10 minutes of hydrogen plasma under a radiofrequency (RF) power of $2 \mathrm{~W}$. It can be seen from Figure 2 (a) that at $2 \mathrm{~W}$, we obtain some nanocrystals which are randomly distributed on the Si substrate. The inset in Figure 2 (a) shows a single Au nanocrystal with facetted sidewalls. We can see from Figure 2 (a) that Au atoms evolve via spinodal dewetting into particles shape rather than a separation into isolated islands. Moreover, one can also see that there is an empty zone around each Au nanocrystal, where no $\mathrm{Au}$ droplet/particle is present. As shown in the cross-section SEM image in Figure 2 (b), the $\mathrm{Au}$ nanocrystals have an irregular shape of a few hundreds of nanometers in height. By 
increasing the RF plasma power from 2 to $5 \mathrm{~W}$, we have successfully grown Au NWs as shown in Figure 2 (c). The inset in Figure 2 (c) shows a zoom of the surface. Figure 2 (d) presents a cross-section view with a single $\mathrm{Au}$ NW, and its top view displaying a hexagonal cross-section in the inset. The facetted NW top end reveals that there is no Au droplet on the NW top. However, this does not mean that there is no Au droplet on top of the NW during its growth. By measuring the top end and bottom end diameter of Au NWs, we found that they are not tapered. The tapering-free of our NWs indicates that the lateral growth does not occur, and that $\mathrm{Au}$ atoms diffuse on the $\mathrm{Au}$ NW sidewall to be incorporated into the top growing surface or droplet. To help understanding the exact function of $\mathrm{H}_{2}$ plasma during $\mathrm{Au}$ NWs, other growth experiments are performed. The first one is as follows. Same growth conditions for $\mathrm{Au}$ NW are performed in a stainless steel PECVD chamber without a-Si:H coating, we observe only the inhibition of Ostwald ripening without growth of Au NW. Note that the melting points of $\mathrm{Au}$ and $\mathrm{Si}$ are $1064{ }^{\circ} \mathrm{C}$ and $1414{ }^{\circ} \mathrm{C}$, whereas their eutectic temperature is only $363{ }^{\circ} \mathrm{C}$. Ostwald ripening of $\mathrm{Au}$ droplets occurs since $\mathrm{Au}$ will interacts with $\mathrm{Si}$ atoms underneath from $\mathrm{Si}$ substrate. However, no extra $\mathrm{Si}$ adatoms can incorporate into $\mathrm{Au} / \mathrm{Si}$ droplet for elongation of one-dimensional nanostructure. The energy of hydrogen ions is not high enough to sputter the Si atoms from the Si substrate. The second experiment is based on the change of a-Si:H coating thickness from 50; 100 (Au NW growth case) to $200 \mathrm{~nm}$ followed by the same Au NW growth conditions. For all the a-Si:H thicknesses, we do observe the growth of Au NWs. In our previous work, ${ }^{23}$ we observed only the inhibition of Ostwald ripening of Au catalyst by applying hydrogen plasma but no Au NWs because only atomic hydrogen and $\mathrm{H}$ ions were involved during the $\mathrm{Au}$ catalyst treatment since the precoating of a-Si:H is not fresh with $\mathrm{SiO}_{\mathrm{x}}$ grown on it (exposed to air at our standard chamber opening temperature at $120{ }^{\circ} \mathrm{C}$ ). These experiments lead us to conclude that running the $\mathrm{H}_{2}$ plasma in an a-Si:H-coated chamber is a key point to successfully grow Au NWs. 

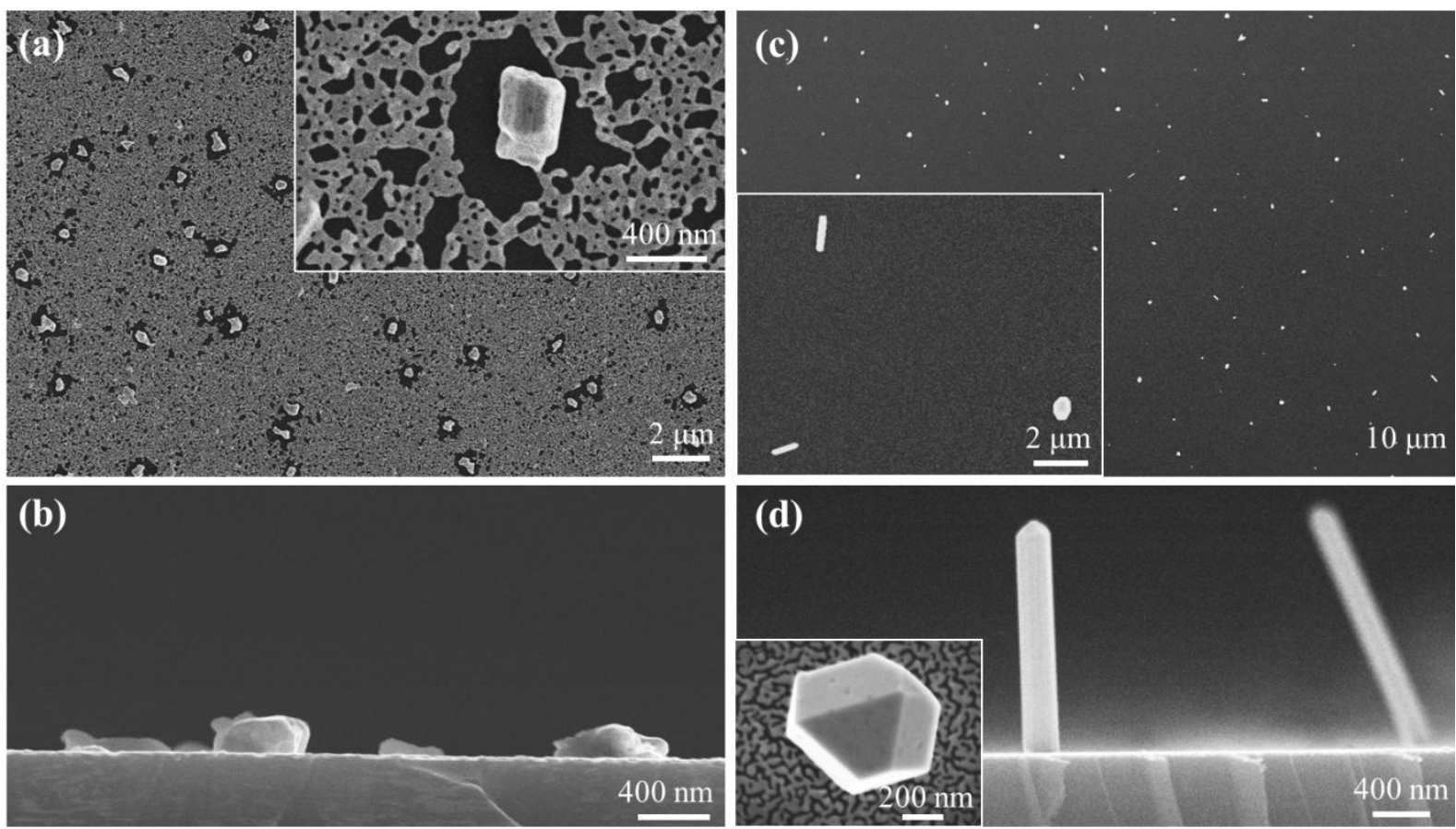

Figure 2. SEM images of VLS-grown Au NWs. (a) Top view and (b) side view of Au NWs grown under a plasma power of $2 \mathrm{~W}$. Inset of (a) shows a zooming. (c) Top view and (d) cross-section view of Au NWs on Si grown under a plasma power of $5 \mathrm{~W}$. The inset of (d) shows a top view image of the NW.

To obtain further information on the properties of the NWs, we first investigated their microstructure in cross section with the help of TEM and high-angle annular dark-field (HAADF) scanning TEM (STEM). Figure 3a shows a low magnification image of a FIB lamella in HAADF-STEM. A higher magnification STEM image is presented in Figure 3 (b) where one can see a weak contrast inside the NW. Two reasons can be attributed to the difference in Z-contrast in STEM image. The first difference comes from the different crystalline quality of several grains, for example, between amorphous and crystalline. The second one is due to the different chemical elements in the sample, for example, between $\mathrm{Au}$ and Si. Note that the signal intensity in STEM image increases with the atomic number producing chemically sensitive Z-contrast. The NW is in [1-10] zone axis showing multiple twinning. The corresponding electron diffraction pattern (EDP) being recorded in that axis 
and parallel to the wire growth axis, the latter was presently [1-10] (Figure 3 (c)). The spots in the EDP correspond to $\mathrm{Au}$, but with a parameter increase of several percent with respect to pure $\mathrm{Au}$, which would indicate the out-of-equilibrium presence of a significant concentration of $\mathrm{Si}$ atoms in solution. In other EDPs, some $\mathrm{Si}$ spots show up indicating the probable presence of Si precipitates. The Red box indicates the region investigated in Figure 3 (d), where a high magnification shows the crystalline lattice of $\mathrm{Au}$ atoms is presented.

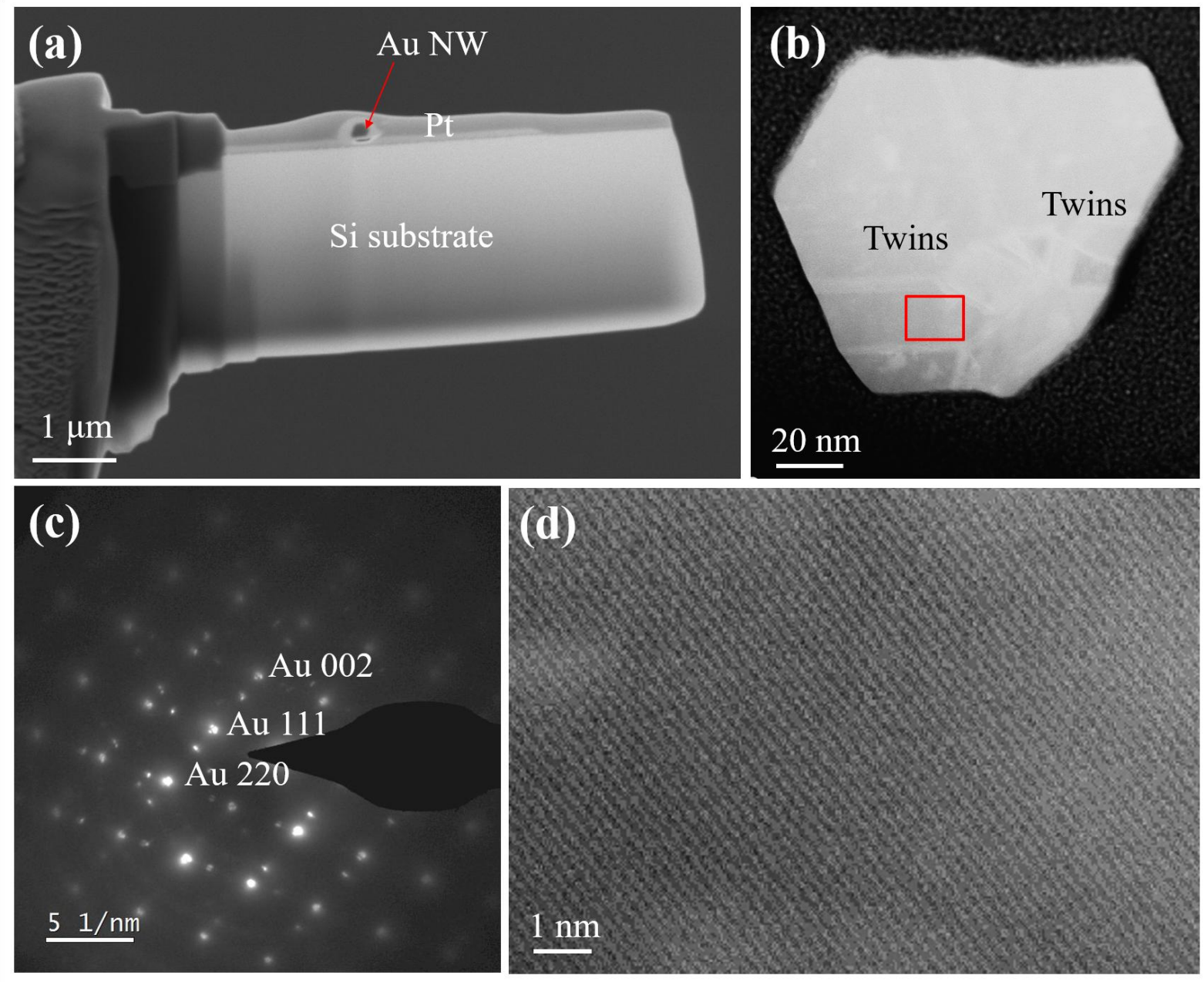

Figure 3. TEM characterization of crystalline quality of Au NW. (a) TEM lamella of a single $\mathrm{Au}$ NW for radical investigation. (b) Low magnification cross-section view of a single $\mathrm{Au}$ NW. The red box indicates the region investigated by high magnification. (c) Corresponding diffraction pattern of Au NW sample. (d) High magnification of an Au NW showing the crystalline lattice of $\mathrm{Au}$ atoms. 
In order to understand the growth mechanism of $\mathrm{Au}$ NWs, let us discuss how the $\mathrm{Au}$ atoms from the evaporated Au thin film can diffuse and form an Au NW. It can be seen from Figure 2 (d) that after NW growth there is no Au droplet on the top end of Si NW. Thus, the first question is whether there was an Au droplet promoting NW growth. In the standard VLS growth mode, one of the main reasons to use a metal liquid catalyst is to favor the formation of a crystalline NW by decreasing the chemical potential difference between the solid NW and liquid droplet. Figure 4 (a) shows a SEM image of a single Au NW. The inset shows the facetted sidewall decorated by Au particles (indicated by arrow). We have found that there is a coverage of Au droplets on the whole sidewall of Au NWs, which should occur after NW growth. If the Au droplets on NW sidewall were formed during NW growth, the size of the $\mathrm{Au}$ droplet on NW top will reduce causing the decrease of NW diameter, which is conflict with our observation with tapering-free $\mathrm{Au}$ NW as presented previously in Figure 2 (d). We have reported in a previous study that a $\mathrm{H}_{2}$ plasma can stabilize the Au catalyst on a Si NW but enhance the diffusion of $\mathrm{Au}$ atoms on the Si NW sidewall once the plasma is switched off. $^{23}$ Therefore, we suggest that an Au droplet could exist on the top of Au NW during NW growth but diffuses on NW sidewall once $\mathrm{H}_{2}$ plasma is switched off.
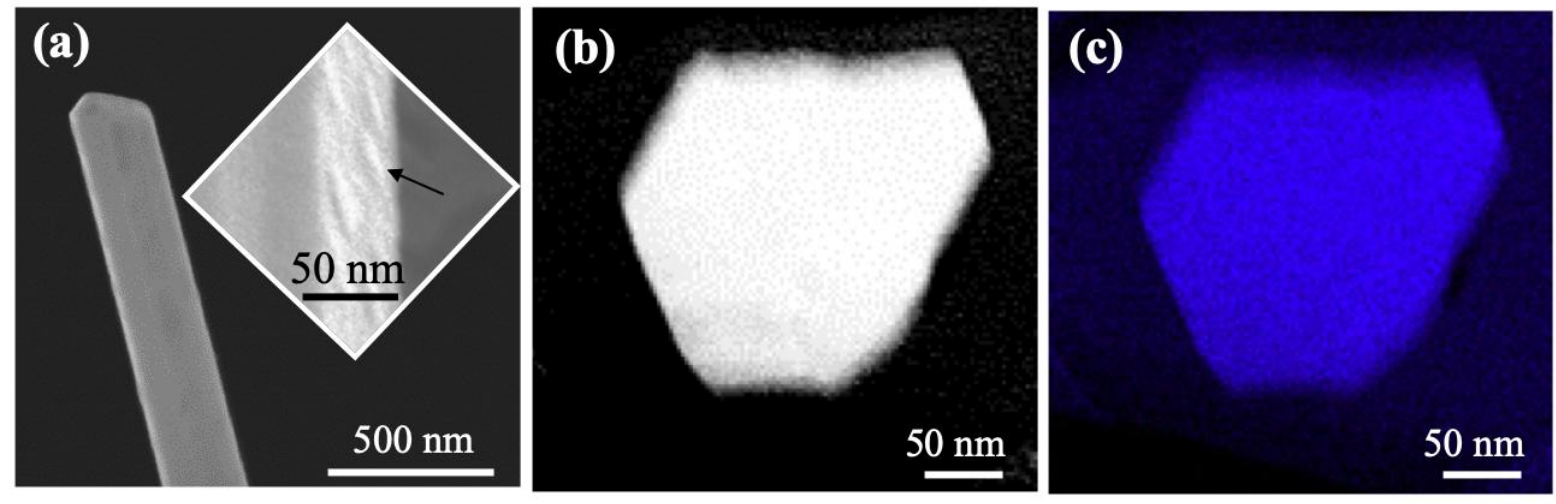

Figure 4. (a) Axial view SEM image. Inset shows the sidewall of NW decorated with Au clusters. (b) BF-STEM images of cross-section viewing of a single Au NW. (c) TEM-EDX mapping of Au atoms in Au NW. 
To support such hypothesis, a cross-section imaging of a NW, namely, a lamella viewing, is desirable. A HAADF-STEM image is presented in Figure 4 (b). The cross-section of the Au NW exhibits a hexagonal shape. The distribution of different elements in Au NW has been investigated by TEM-EDX. According to the analysis of TEM-EDX, Au atoms are identified. It can be seen from the TEM-EDX mapping that Au atoms are distributed homogeneously and no visible segregation is detected. Therefore, we conclude that Au atoms exhibit a homogenous radial distribution. By combing TEM-EDX image in Figure 4 (c) and high magnification viewing in STEM mode (Figure 3 (b)), we have been able to confirm that the weak contrast in Figure 3 (b) comes from the different crystalline quality of Au NW. Note that there is an overlap between $\mathrm{Si}-\mathrm{K}$ and $\mathrm{Au}-\mathrm{M}$ lines in EDX mapping, therefore, it is difficult to quantitatively distinguish whether there are Si atoms in Au NWs. Let us recall that the EDP (Figure 3 (c)) indicates a parameter increase of several percent, which suggests the presence of $\mathrm{Si}$ in the Au lattice. To investigate the microstructure orientation of $\mathrm{Au} \mathrm{NWs,}$ TKD has been used. A SEM image of the side view of an Au NW is presented in Figure 5. As in the case of STEM view (Figure 3 (b)), we observe some contrast from SEM image. The corresponding TKD orientation mappings of $\mathrm{Au} \mathrm{NW}$ along $\mathrm{X}, \mathrm{Y}$ and $\mathrm{Z}$ directions are presented in Figure 5 (a), (b) and (c), respectively. Different colors in TKD mapping indicate their different orientations. From TKD mapping, we could conclude that Au NWs consists of several grains with different orientation. 

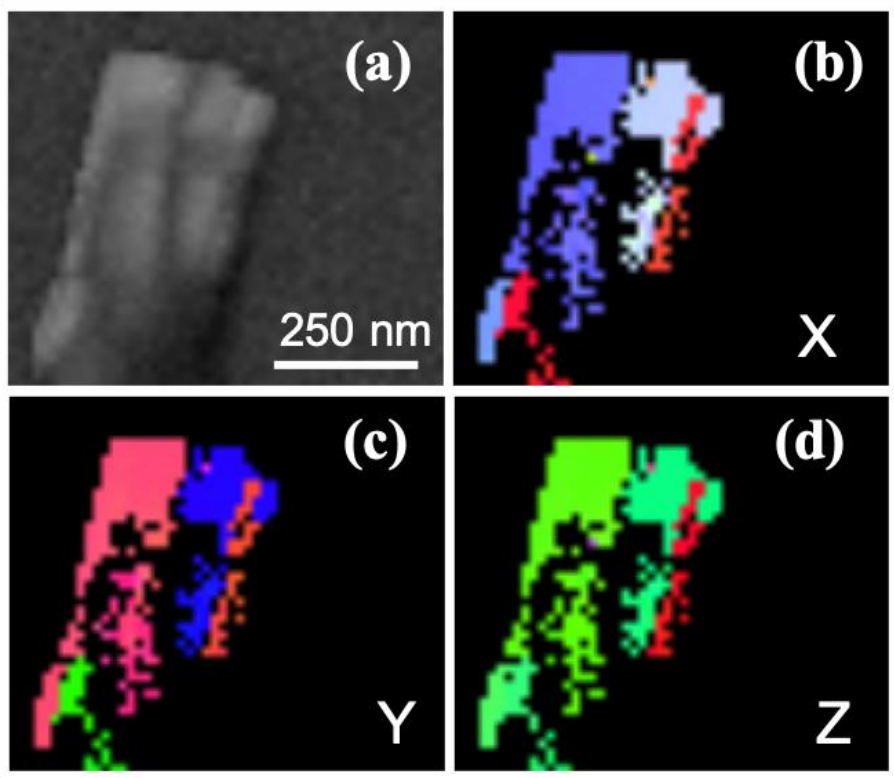

Figure 5. (a) Side view SEM image of Au NW. TKD orientation mapping of the Au NW along (b) X; (c) Y and (d) Z direction.

Metal NWs, especially Au, have been widely used in SERS measurements ${ }^{15,24,25}$ due to their strong localized surface plasmon resonance (LSPR). ${ }^{26}$ Figure 6 (a) shows an optical image of $\mathrm{Au}$ NWs appearing as dark spots. In order to exploit the huge local field amplification of Raman light scattered from molecules adsorbed on nanostructured $\mathrm{Au}$ surfaces,${ }^{27}$ we covered our sample with an organic dye molecule, namely methylene blue (MB) $10^{-4} \mathrm{M}$ (see methods). The main characteristic Raman bands of MB are at $446 \mathrm{~cm}^{-1}$ and 1624 $\mathrm{cm}^{-1}$, due to the $\mathrm{C}-\mathrm{N}-\mathrm{C}$ skeletal bending and the $\mathrm{C}-\mathrm{C}$ stretching, respectively. ${ }^{28}$ In Figure 6 (b) we can observe the SERS map of the MB vibrational mode at $1624 \mathrm{~cm}^{-1}$ acquired from the same region showed Figure 6 (a), whose comparison with SEM images highlights a correlation between the brighter spots (high SERS intensity) and the presence of Au NWs. The white arrows in Figure 6 (b) indicates the positions correspondent to the SERS spectra presented in Figure 6 (c) where the green line represent the SERS intensity when the light is collected from the NWs position, i.e. black structures in Figure 6 (a), and far from NWs (black line), where some Au nanoparticles (NPs) are also present. Both spectra are taken in 
the same experimental conditions and both of them features the characteristic peaks of $\mathrm{MB}$, but the Au NWs ensure a signal stronger about 27 times compared to Au NPs. A very similar picture is observed switching the excitation wavelength at $633 \mathrm{~nm}$ with a similar intensity contrast $(\approx 30)$ between SERS on Au NWs and SERS on Au NPs (Figure 6 (d)). The main difference between the two-excitation configuration is related to the different enhancement of the peak at $1624 \mathrm{~cm}^{-1}$ compared to the one at $446 \mathrm{~cm}^{-1}$. In the first case $\left(\lambda_{\text {exc }}=532 \mathrm{~nm}\right)$, $I_{1624} / I_{446} \approx 10$, while for $\left.\lambda_{e x c}=633 \mathrm{~nm}\right), I_{1624} / I_{446} \approx 1$. This effect is due to the spectral dependence of the plasmonic properties of $\mathrm{Au}$ NWs, which is reflected by the different amplification of Raman peaks in different spectral position ${ }^{29}$.

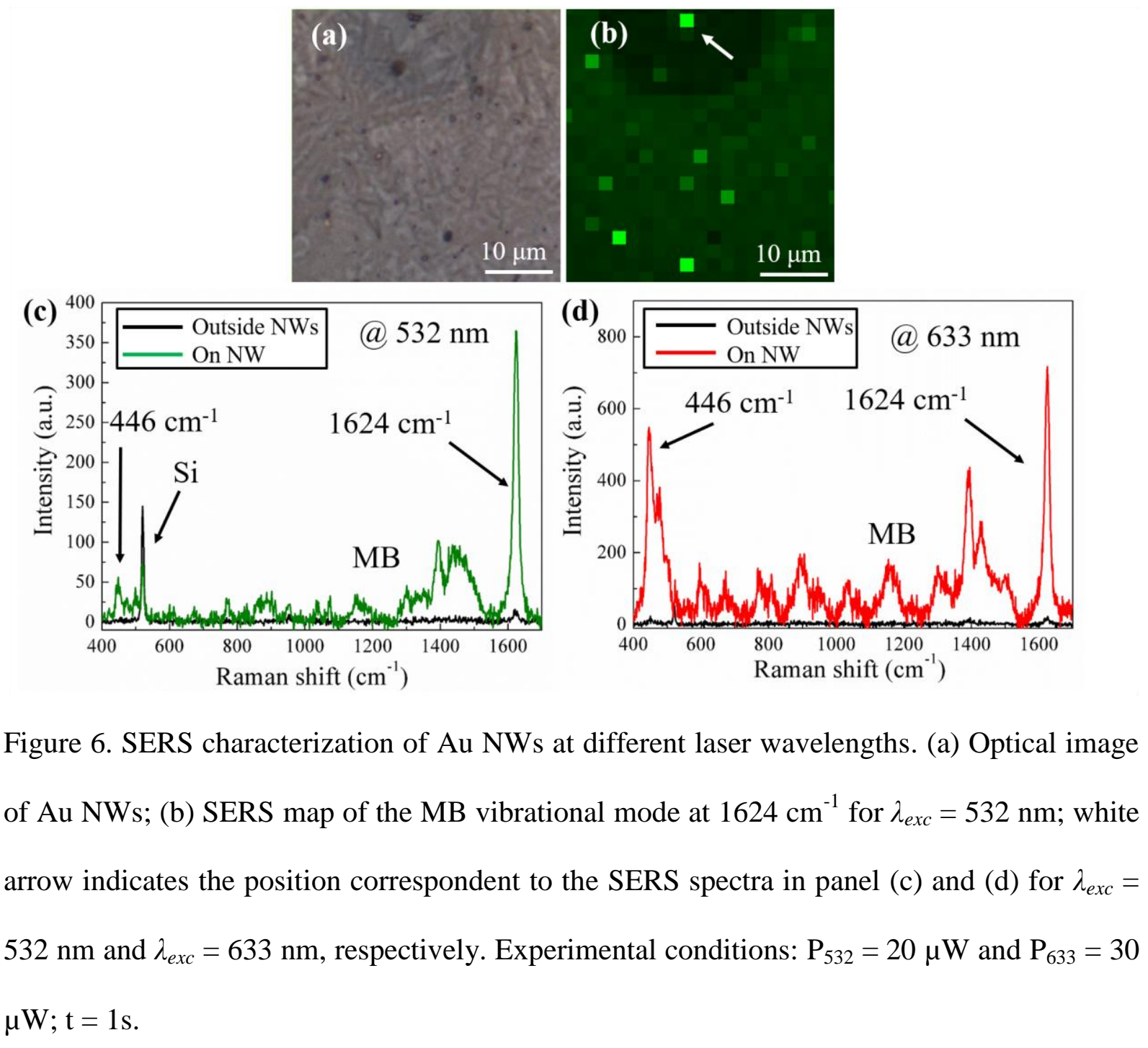


We now perform laser-induced photocurrent measurements on Au NWs-covered Si wafer under different laser excitation conditions, including controls of the baseline current level as defined by the recording micropipette (i.e., the current under dark condition) and the pulse width and laser power from the light source ${ }^{30}$ The light pulse was delivered through a microscope objective while recording changes of the ionic current. The detailed photocurrent measurement setup is presented in the Methods parts. ${ }^{30}$ Before we measure the photocurrent in the presence of Au NWs, we measured that generated by the native (111) Si wafer. The current response is presented in Figure 7 (a). Under laser excitation (wavelength, $532 \mathrm{~nm}$ ), we observed that the recorded photocurrent polarity changed from positive to negative sign when the pipette baseline current is switched from $+3 \mathrm{nA}$ to $-3 \mathrm{nA}$. Moreover, there is no photoresponse when the baseline current is hold at $0 \mathrm{nA}$. These together suggest that the ntype (111) Si wafer itself yield only negligible photocurrent. With Au NWs grown on the wafer, we observed several major changes (Figure 7 (b)). First, we recorded photocurrent with much larger amplitude (Figure 7 (c)). The current is primarily capacitive, showing a negative and a positive peak at the onset and the end of the light pulse, respectively. The negative peak is suggestive of a photoanodic affect, and vice versa. Second, the photocurrent doesn't change its sign when the polarity of the photocurrent baseline is flipped, and there is a pronounced photocurrent recorded at zero baseline current level (Figure 7 (c)). Finally, the temporal distance between the two capacitive peaks can be readily tuned by changing the duration of the light pulses, as shown in the current traces recorded under at $0 \mathrm{nA}$ baseline level and variable pulse width from 1 to 5 and to $10 \mathrm{~ms}$, by keeping the power at (Figure 7 (d)). Overall, these results suggest that the photoelectrochemical effect from the c-Si wafer can be significantly enhanced with the as-grown Au nanostructures on its surface ${ }^{30}$. This property can be potentially leveraged for applications such as photoelectrochemical modulation of biological activities ${ }^{31}$. Since there is no response from the c-Si wafer when the injection 
current is $0 \mathrm{nA}$, the following measurement is performed at $0 \mathrm{nA}$ current injection by varying the pulse width from 1 to 5 and to $10 \mathrm{~ms}$, by keeping the power at $\sim 56 \mathrm{~mW}$ (Figure 7 (d)).
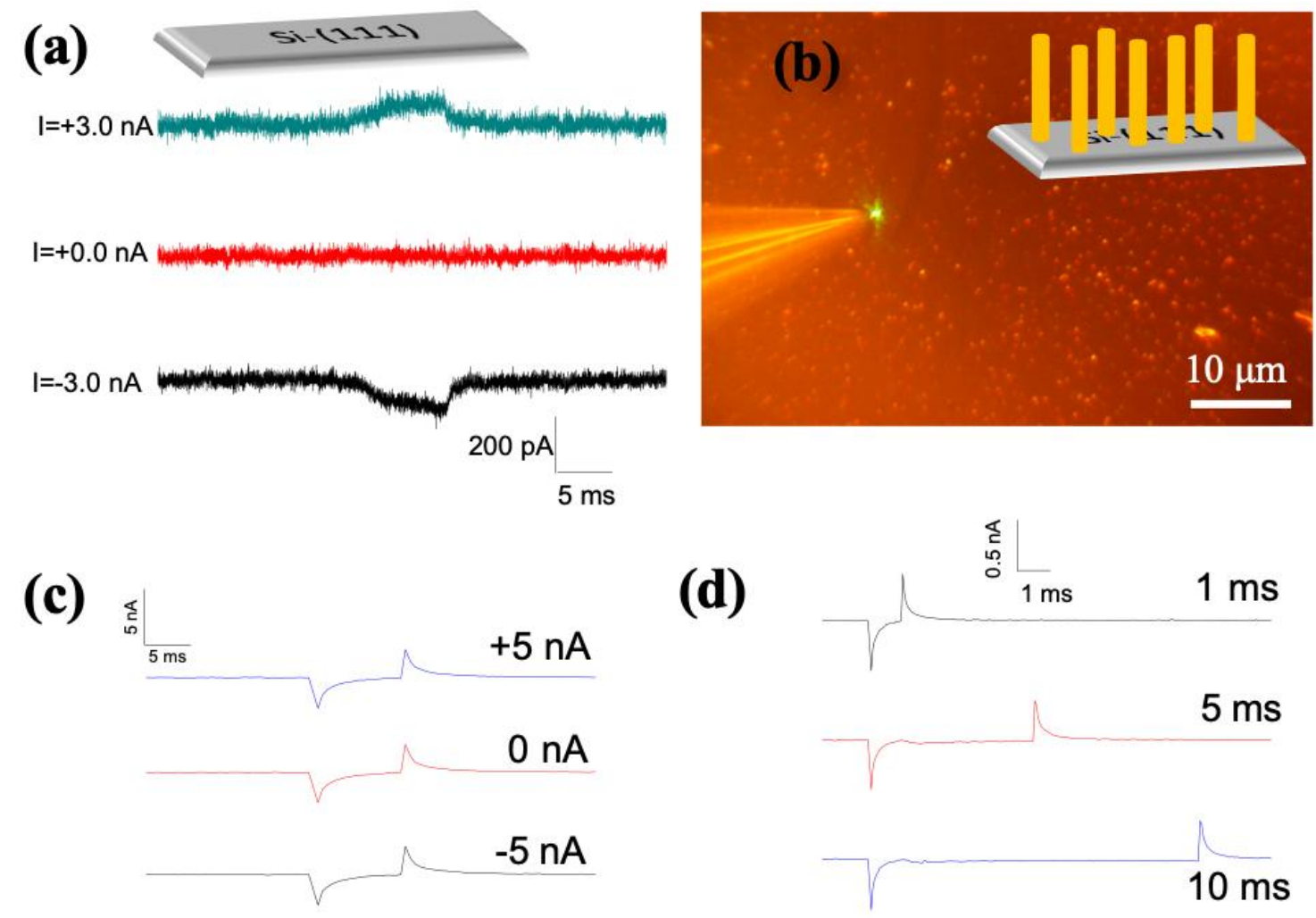

(d)

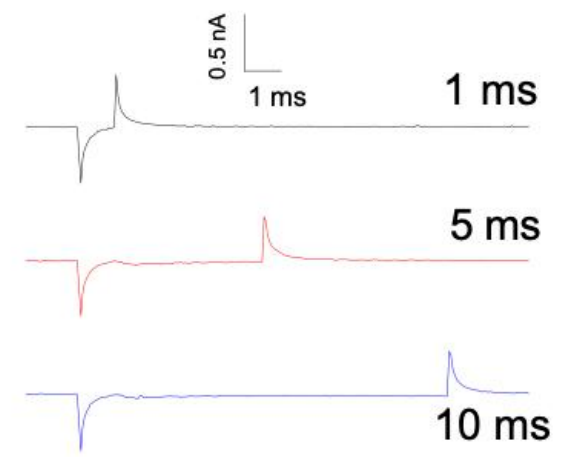

Figure 7. Photocurrent measurements from a reference c-Si wafer and from Au NWs under different laser conditions. (a) Measurements on a (111) n-type c-Si wafer; (b) Optical image of the laser spot on an Au NW; (c) Variation in holding level from -5 ; 0 ; to $+5 \mathrm{nA}$ by keeping the laser power at $\sim 56 \mathrm{~mW}$ and pulse width of $5 \mathrm{~ms}$; (d) Variation in pulse width from 1to 5 and to $10 \mathrm{~ms}$ while keeping the laser power at $\sim 56 \mathrm{~mW}$ and the holding level at $0 \mathrm{nA}$.

In order to discuss the transport of $\mathrm{Au}$ atoms, required to explain the growth mechanism of Au NWs, it is very important to know the dependence of Au NW growth rate on NW diameter, since this allows us to analyze the main incorporation pathway of Au atoms. As concluded from Figure 2, it is plausible to assume that the growing interface is at the top end of Au NWs. Therefore, we considered that our Au NWs follow a surface diffusion growth 
mode. The length (L) and diameter (D) of Au NWs are measured from cross-section SEM images. The growth rate $(\mathrm{dL} / \mathrm{dt})$ is then calculated by dividing the NW length by the effective growth time t. The dependence of $\mathrm{dL} / \mathrm{dt}$ on $\mathrm{D}$ is plotted as open squares in Figure 8 (a), revealing a $\mathrm{dL} / \mathrm{dt} \sim 1 / \mathrm{D}$ dependence, where the dominant incorporation pathway of $\mathrm{Au}$ atoms is via the NW sidewall diffusion of $\mathrm{Au}$ atoms. By taking into account the mass conservation of $\mathrm{Au}$ atoms and Nernst-Einstein's diffusion flux expression, we can write down the following equation $^{23}$ :

$$
\frac{d L}{d t}=\Psi \frac{\Delta \mu}{d} \quad \text { Eq. } 1
$$

where, $\Psi=4 \Omega n D / k_{B} T \lambda, \Omega$ is the specific volume of Au, $n$ is the Au adatom density at NW sidewall, which is taken as its maximum value (one monolayer) with a value of $\sim 1 \times 10^{19} \mathrm{~m}^{-2}$. The effective supersaturation of Au atoms on NW sidewall with respect to the $\operatorname{droplet} \Delta \mu / k_{B} T$ equals to $2^{23}$. The diffusion length of Au adatoms depends on the surface state, which is a very complex system including $\mathrm{Si}$ atoms, Au atoms, crystalized $\mathrm{Au}$ and surrounding gas and plasma environment (ions, particles, radicals, etc.). To the best of our knowledge, the precise value of the diffusion length of $\mathrm{Au}$ atoms on such systems is unknown. However, from radial distribution function, we can estimate roughly the value of the diffusion length. The diffusion length $\lambda$ is estimated to be $7.3 \mu \mathrm{m}$ at our NW growth temperature $\left(475^{\circ} \mathrm{C}\right)$, which equals to the sum of two contributing paths: the longest NW length grown in this work $(2.3 \mu \mathrm{m})$ and the half average distance between two adjacent Au NWs $(5 \mu \mathrm{m})$. The average distance between $\mathrm{Au}$ NWs is calculated from the radial distribution function of Au NWs (obtained by using ImageJ) as presented in Figure 8 (b). The long Au atom collection distance is also the reason for no lateral growth (one of the tapering-free reasons discussed previously). The distribution curve exhibits a flat form starting from $10 \mu \mathrm{m}$, which indicates that Au NWs are randomly distributed. A fitting curve is plotted in Figure 8 (a), yielding $D$ (diffusivity of Au adatoms on 
$\mathrm{Au}$ NW sidewall) to be $2.56 \times 10^{-8} \mathrm{~cm}^{2} / \mathrm{s}$. The reasons attributed to the random distribution of Au NWs can be discussed as follows. The Au thin film under hydrogen plasma follows the spinodal dewetting with random distribution of Au droplets (size and position). Au droplets with different diameters will cause the diameter of each NW to be different and the random distribution of Au droplets will induce the random position of Si NWs.
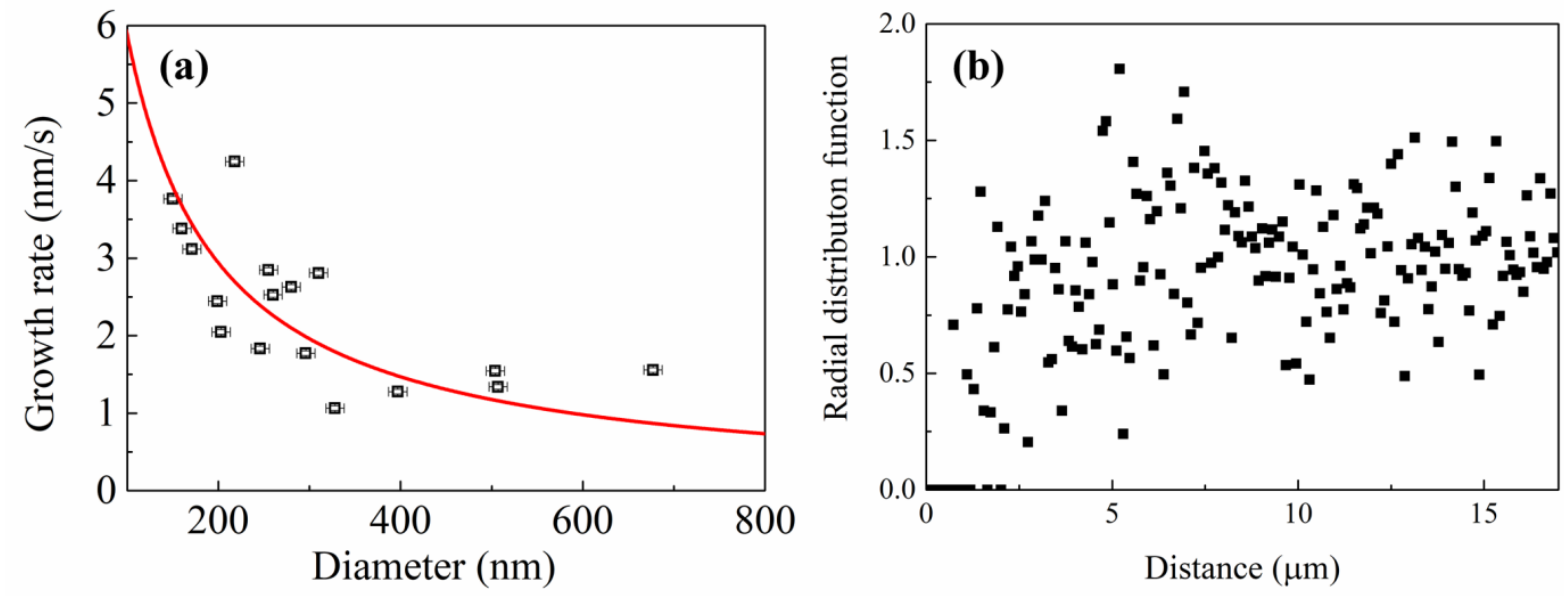

Figure 8. (a) Evolution of NW growth rate on NW diameter. Red curve presents the fitting based on Eq. 1. (b) Radial distribution functions of Au NWs.

Let us now discuss the growth mechanism of Au NWs. Once the c-Si substrate covered with $5 \mathrm{~nm}$ of $\mathrm{Au}$ thin film is exposed to a $\mathrm{H}_{2}$ plasma, there are two main effects influencing the growing system. The first one is the effect on Au thin film. The Au thin film follows Ostwald ripening after heating the sample to $475{ }^{\circ} \mathrm{C}$ without $\mathrm{H}_{2}$ plasma. However, this Ostwald ripening can be inhibited by applying a $\mathrm{H}_{2}$ plasma ${ }^{23}$. The Au thin film then follows a spinodal dewetting as shown in Figure 2 (a). In this case, the Au thin film evolves towards a mesh shape rather than isolated Au droplets. The second effect of $\mathrm{H}_{2}$ plasma is its interaction with a-Si:H-coated reactor chamber walls. During $\mathrm{H}_{2}$ plasma treatment, atomic hydrogen can etch the a-Si:H from the wall ${ }^{32}$. The etched $\mathrm{Si}$ can be re-deposited on the samples, ${ }^{33}$ in particularly at the surface of Au thin film (mesh shape) and incorporate into Au 
thin film to form Si/Au alloy at our growth temperature $\left(475^{\circ} \mathrm{C}\right)$. The interaction of $\mathrm{Au}$ adatoms and Si radicals is a key point to successfully grow Au NWs since no Au NW can be grown without the chamber coating of a-Si:H. The eutectic temperature of the $\mathrm{Si} / \mathrm{Au}$ alloy system is $363{ }^{\circ} \mathrm{C}$. Since our growth temperature is above the eutectic temperature, the diffusivity of $\mathrm{Au}$ adatoms and Si radicals can be greatly enhanced. The supersaturation in $\mathrm{Au}$ droplet will increase with the decrease of the droplet diameter because of the GibbsThomson's effect. Therefore, Au droplets with a diameter larger than a certain value (e.g. 200 $\mathrm{nm}$ in this work) can reach the supersaturation and start the nucleation earlier. Once nucleation has started, the surrounding Au droplets (within a given range) cannot form new nucleation sites since the energy barrier to crystallize on a crystalline seed is lower than that to form a new nucleation seed. However, for Au droplets at distances longer than the diffusion length of $\mathrm{Au}$ atoms (e.g. $5 \mu \mathrm{m}$ in this work), new nucleation events take place in $\mathrm{Au}$ droplets with the right diameter. As a consequence, sparse Au NWs can be obtained. The continuous Au thin film with mesh shape is the critical element to grow such kind of Au NWs. Indeed, if the inhibition of Oswald ripening is not efficient, for example in the case of low hydrogen plasma powder $(2 \mathrm{~W})$, Au droplets with large diameters are separated from the Au mesh thin film as shown in Figure 2 (a). The diffusion of $\mathrm{Au}$ atoms to the Au droplets will be blocked by the zone without the coverage of Au thin film. In this case, only very short Au nanocrystals can be grown.

\section{Conclusion}

To conclude, we have developed an efficient maskless method to grow Au NWs with the help of a $\mathrm{H}_{2}$ plasma. By properly choosing the plasma power, we have successfully changed the growth process from Au nanocrystals to Au NWs. SEM investigation shows that these $\mathrm{Au}$ NWs are tapering-free. From TEM-EDX mapping, we show that $\mathrm{Au}$ atoms have a homogeneous radial distribution in Au NWs. EDP and HRTEM shows the existence of Si 
atoms in Au NWs. Compared to the adjacent Au NPs, Au NWs feature a stronger near-field enhancement in the optical spectral range, as evidenced by the SERS intensity scattered by MB molecules adsorbed at the nanostructures surface, which is about 30 times higher in the second case. In addition, this characterization may allow to spot the presence of AuNWs without the necessity of SEM analysis. Strong laser-induced photocurrent can be generated from Au NWs without polarity dependence on current injection. We observed a dependence of the growth rate as a function of diameter (dL/dt 1/D). This is attributed to the formation of an Au layer with mesh shape. Our method can pave the way to fabricate metal NWs and can be easily extended to other types of metal NWs by combing their thin films and $\mathrm{H}_{2}$ plasma treatment.

\section{Experimental Section}

\section{TEM lamellar preparation}

The standard lamellar preparation method based on focused ion beam (FIB) was used in this work. The "out-of-plane" Au NWs are randomly scratched to "in-plane" ones by a diamond pen. A Pt protective layer is then deposited on these "in-plane" NWs to avoid the implantation damage during FIB process. Note that the first ten manometers of Pt layer are deposited via electron beam-induced deposition (EBID), then followed by a faster deposition via ion beaminduced deposition FIB (IBID). The purpose of this two-mode-deposition is to avoid the contamination of NW surface caused by Ga ions either from IBID or from FIB ion milling. Then, the Si chunk containing Au NW is lifted out and transferred to a $\mathrm{Cu}$ lamellar support. Finally, the TEM support is load into our TEM platform.

\section{TKD sample preparation}

The TKD sample preparation consists of two steps. The first step is to scratch Au NWs onto a native Si wafer as in the case of TEM lamellar sample preparation. Then, a single"in-plane" 
Au NW can be picked up with a W tip under optical microscopy, where the sharp W tip is prepared by electro polishing.

\section{SERS measurements}

SERS activity of AuNWs was studied coating the nanowires surface with methylene blue (MB), an organic dye with a strong affinity with Au. The sample was immersed in a watery solution of MB $10^{-4} \mathrm{M}$ for two hours. Afterwards it was rinsed in water in order to remove the excess molecules not bounded to the Au surface and then dried in air.

Spectroscopic measurements were performed using a LabRAM HR800 micro spectrometer (Horiba Scientific) equipped with a solid-state laser and an HeNe laser emitting at $532 \mathrm{~nm}$ and $633 \mathrm{~nm}$, respectively. The incident beam was conveyed on the sample surface with a BXFM Olympus microscope via a 100X magnification objective $(\mathrm{NA}=0.9)$. The same path was used to collect SERS signal in a backscattering configuration and finally recorded by a CCD detector (Synapse - Horiba Scientific).

\section{Photocurrent measurements}

Photocurrent measurements were performed via the method of single channel voltage clamped recording. This was done using a patch-clamp amplifier and digitizer (Axopatch 200B). The wafer with Au NWs was immersed in 1X PBS (Fisher Scientific) in a petridish. The top of the vertical NWs was focused on using a water immersion lens (20X/0.5 NA) on an upright microscope (Olympus BX61WI). Light pulses were delivered through this lens onto Au NWs. Light pulses were generated using a $532 \mathrm{~nm}$ laser (Laser glow, diode -pumped solid state laser, $\sim 5 \mu \mathrm{m}$ spot size) or $515 \mathrm{~nm}$ LED (Thorlabs, $\sim 500 \mu \mathrm{m}$ spot size). Pulses were delivered to these light sources using a digitizer (Molecular devices, Digidata 1550). For recording the photoresponses, glass pipettes (OD: $1.5 \mathrm{~mm}$, ID: $1.10 \mathrm{~mm}$, Length: $10 \mathrm{~cm}$, Sutter instruments Cat No: BF150-110-10) of $\sim 1 \mathrm{M} \Omega$ were pulled (Sutter Instrument, P-97) 
and their micro-tip lowered and focused on the tip of the Au NW (Gap of $\sim 2 \mu \mathrm{m}$ ) before the stimulation pulse is applied. A bias of $-0.5 \mathrm{~V}$ in parallel with the stimulation pulse at various holding current levels or current injection as the photoresponse recording was done. The data were analyzed and the origin of photo response elucidated by a previously developed method. ${ }^{30}$ For concluding on the photothermal measurements on pure native (111) n-type Si wafer, the same pipette used for recording was calibrated for its resistance as a function of temperature. For pipette calibration, it was dipped in pre-heated $\mathrm{PBS}$ at approximately $50^{\circ} \mathrm{C}$ and allowed to cool down in air as the resistance was recorded alongside temperature with a thermocouple.

\section{Acknowledgments}

A. Foti gratefully recognize funding within 16-CE09-0029-03 TIPTOP_1 and 17-CE240005-04 TRAMP ANR projects.

\section{Author contributions}

W.C. R.G. and P.R. designed and conducted the experiments, analyzed the data, wrote the main text and prepared the figures. J.L.M. and S.M. performed the TEM experiments. E.A. and C.K. perormed the TKD measurments. E.A. prepared the TEM lamellars. V.N. and B.T. performed laser-induced photocurrent measurements. A.F. concucted SERS measurements. All authors participated in discussing and reviewing the manuscript.

\section{References}

(1) Wagner, R. S.; Ellis, W. C., Vapor Liquid Solid mechanism of single crystal growth. Applied Physics Letters 1964, 4, (5), 89-90.

(2) Morales, A. M.; Lieber, C. M., A Laser Ablation Method for the Synthesis of Crystalline Semiconductor Nanowires. Science 1998, 279, (5348), 208-211.

(3) Tian, B.; Lieber, C. M., Nanowired Bioelectric Interfaces. Chemical Reviews 2019, 119, (15), 9136-9152.

(4) Chen, W.; Roca i Cabarrocas, P., Rational design of nanowire solar cells: from single nanowire to nanowire arrays. Nanotechnology 2019, 30, (19), 194002. 
(5) Tian, B.; Zheng, X.; Kempa, T. J.; Fang, Y.; Yu, N.; Yu, G.; Huang, J.; Lieber, C. M., Coaxial silicon nanowires as solar cells and nanoelectronic power sources. Nature 2007, 449, (7164), 885-889.

(6) Chen, W.; Yu, L.; Misra, S.; Fan, Z.; Pareige, P.; Patriarche, G.; Bouchoule, S.; Roca i Cabarrocas, P., Incorporation and redistribution of impurities into silicon nanowires during metal-particle-assisted growth. Nature Communications 2014, 5, 4134.

(7) Chen, W.; Pareige, P.; Castro, C.; Xu, T.; Grandidier, B.; Stiévenard, D.; Roca i Cabarrocas, P., Atomic characterization of Au clusters in vapor-liquid-solid grown silicon nanowires. Journal of Applied Physics 2015, 118, (10), 104301.

(8) Fang, Y.; Jiang, Y.; Cherukara, M. J.; Shi, F.; Koehler, K.; Freyermuth, G.; Isheim, D.; Narayanan, B.; Nicholls, A. W.; Seidman, D. N.; Sankaranarayanan, S. K. R. S.; Tian, B., Alloy-assisted deposition of three-dimensional arrays of atomic gold catalyst for crystal growth studies. Nature Communications 2017, 8, (1), 2014.

(9) Luo, Z.; Jiang, Y.; Myers, B. D.; Isheim, D.; Wu, J.; Zimmerman, J. F.; Wang, Z.; Li, Q.; Wang, Y.; Chen, X.; Dravid, V. P.; Seidman, D. N.; Tian, B., Atomic gold-enabled three-dimensional lithography for silicon mesostructures. Science 2015, 348, (6242), 1451-1455.

(10) Basarir, F.; Irani, F. S.; Kosemen, A.; Camic, B. T.; Oytun, F.; Tunaboylu, B.; Shin, H. J.; Nam, K. Y.; Choi, H., Recent progresses on solution-processed silver nanowire based transparent conducting electrodes for organic solar cells. Materials Today Chemistry 2017, 3, 60-72.

(11) Jin, Y.; Sun, Y.; Wang, K.; Chen, Y.; Liang, Z.; Xu, Y.; Xiao, F., Long-term stable silver nanowire transparent composite as bottom electrode for perovskite solar cells. Nano Research 2018, 11, (4), 1998-2011.

(12) Russell, C.; Welch, K.; Jarvius, J.; Cai, Y.; Brucas, R.; Nikolajeff, F.; Svedlindh, P.; Nilsson, M., Gold Nanowire Based Electrical DNA Detection Using Rolling Circle Amplification. ACS Nano 2014, 8, (2), 1147-1153.

(13) Wu, Y.; Ali, M. R. K.; Dong, B.; Han, T.; Chen, K.; Chen, J.; Tang, Y.; Fang, N.; Wang, F.; El-Sayed, M. A., Gold Nanorod Photothermal Therapy Alters Cell Junctions and Actin Network in Inhibiting Cancer Cell Collective Migration. ACS nano 2018, 12, (9), 9279-9290.

(14) Kang, X.; Guo, X.; Niu, X.; An, W.; Li, S.; Liu, Z.; Yang, Y.; Wang, N.; Jiang, Q.; Yan, C.; Wang, H.; Zhang, Q., Photothermal therapeutic application of gold nanorods-porphyrin-trastuzumab complexes in HER2-positive breast cancer. Scientific Reports 2017, 7, 42069.

(15) Hu, Y.; Cheng, H.; Zhao, X.; Wu, J.; Muhammad, F.; Lin, S.; He, J.; Zhou, L.; Zhang, C.; Deng, Y.; Wang, P.; Zhou, Z.; Nie, S.; Wei, H., Surface-Enhanced Raman Scattering Active Gold Nanoparticles with Enzyme-Mimicking Activities for Measuring Glucose and Lactate in Living Tissues. ACS Nano 2017, 11, (6), 5558-5566.

(16) Lee, J.-H.; Cheglakov, Z.; Yi, J.; Cronin, T. M.; Gibson, K. J.; Tian, B.; Weizmann, Y., Plasmonic Photothermal Gold Bipyramid Nanoreactors for Ultrafast Real-Time Bioassays. Journal of the American Chemical Society 2017, 139, (24), 8054-8057.

(17) Menke, E. J.; Thompson, M. A.; Xiang, C.; Yang, L. C.; Penner, R. M., Lithographically patterned nanowire electrodeposition. Nature Materials 2006, 5, 914.

(18) Jiang, X.; Qiu, X.; Fu, G.; Sun, J.; Huang, Z.; Sun, D.; Xu, L.; Zhou, J.; Tang, Y., Highly simple and rapid synthesis of ultrathin gold nanowires with (111)-dominant facets and enhanced electrocatalytic properties. Journal of Materials Chemistry A 2018, 6, (36), 17682-17687.

(19) Jana, N. R.; Gearheart, L.; Murphy, C. J., Seed-Mediated Growth Approach for ShapeControlled Synthesis of Spheroidal and Rod-like Gold Nanoparticles Using a Surfactant Template. Advanced Materials 2001, 13, (18), 1389-1393.

(20) Jana, N. R., Gram-Scale Synthesis of Soluble, Near-Monodisperse Gold Nanorods and Other Anisotropic Nanoparticles. Small 2005, 1, (8 - 9), 875-882.

(21) Billot, L.; Lamy de la Chapelle, M.; Grimault, A. S.; Vial, A.; Barchiesi, D.; Bijeon, J. L.; Adam, P. M.; Royer, P., Surface enhanced Raman scattering on gold nanowire arrays: Evidence of strong multipolar surface plasmon resonance enhancement. Chemical Physics Letters 2006, 422, (4), 303307. 
(22) Dawson, K.; Strutwolf, J.; Rodgers, K. P.; Herzog, G.; Arrigan, D. W. M.; Quinn, A. J.; O’Riordan, A., Single Nanoskived Nanowires for Electrochemical Applications. Analytical Chemistry 2011, 83, (14), 5535-5540.

(23) Chen, W.; Roca i Cabarrocas, P., Insights into gold-catalyzed plasma-assisted CVD growth of silicon nanowires. Applied Physics Letters 2016, 109, (4), 043108.

(24) D’Andrea, C.; Fazio, B.; Gucciardi, P. G.; Giordano, M. C.; Martella, C.; Chiappe, D.; Toma, A.; Buatier de Mongeot, F.; Tantussi, F.; Vasanthakumar, P.; Fuso, F.; Allegrini, M., SERS Enhancement and Field Confinement in Nanosensors Based on Self-Organized Gold Nanowires Produced by lonBeam Sputtering. The Journal of Physical Chemistry C 2014, 118, (16), 8571-8580.

(25) Cara, E.; Mandrile, L.; Ferrarese Lupi, F.; Giovannozzi, A. M.; Dialameh, M.; Portesi, C.; Sparnacci, K.; De Leo, N.; Rossi, A. M.; Boarino, L., Influence of the long-range ordering of gold-coated Si nanowires on SERS. Scientific Reports 2018, 8, (1), 11305.

(26) Maier, S. A., Plasmonics: Fundamentals and Applications. ed.; Springer US: 2007.

(27) Le Ru, E. C.; Etchegoin, P. G., Principles of Surface-Enhanced Raman Spectroscopy. ed.; Elsevier: 2009.

(28) Naujok, R. R.; Duevel, R. V.; Corn, R. M., Fluorescence and Fourier Transform surfaceenhanced Raman scattering measurements of methylene blue adsorbed onto a sulfur-modified gold electrode. Langmuir 1993, 9, (7), 1771-1774.

(29) D’Andrea, C.; Irrera, A.; Fazio, B.; Foti, A.; Messina, E.; Maragò, O. M.; Kessentini, S.; Artoni, P.; David, C.; Gucciardi, P. G., Red shifted spectral dependence of the SAB. Journal of Optics 2015, 17, (11), 114016.

(30) Jiang, Y.; Li, X.; Liu, B.; Yi, J.; Fang, Y.; Shi, F.; Gao, X.; Sudzilovsky, E.; Parameswaran, R.; Koehler, K.; Nair, V.; Yue, J.; Guo, K.; Fang, Y.; Tsai, H.-M.; Freyermuth, G.; Wong, R. C. S.; Kao, C.-M.; Chen, C.-T.; Nicholls, A. W.; Wu, X.; Shepherd, G. M. G.; Tian, B., Rational design of silicon structures for optically controlled multiscale biointerfaces. Nature Biomedical Engineering 2018, 2, (7), 508-521. (31) Parameswaran, R.; Carvalho-de-Souza, J. L.; Jiang, Y.; Burke, M. J.; Zimmerman, J. F.; Koehler, K.; Phillips, A. W.; Yi, J.; Adams, E. J.; Bezanilla, F.; Tian, B., Photoelectrochemical modulation of neuronal activity with free-standing coaxial silicon nanowires. Nature Nanotechnology 2018, 13, (3), 260-266.

(32) Fontcuberta i Morral, A.; Roca i Cabarrocas, P., Role of hydrogen diffusion on the growth of polymorphous and microcrystalline silicon thin films. Eur. Phys. J. Appl. Phys. 2006, 35, (3), 165-172.

(33) Kail, F.; Hadjadj, A.; Roca i Cabarrocas, P., Hydrogen diffusion and induced-crystallization in intrinsic and doped hydrogenated amorphous silicon films. Thin Solid Films 2005, 487, (1), 126-131. 